\title{
Irritable Baby With Weight Loss and a Periorificial and Truncal Rash
}

\author{
Katherine G. Cvancara, BA; Joseph L. Cvancara, MD
}
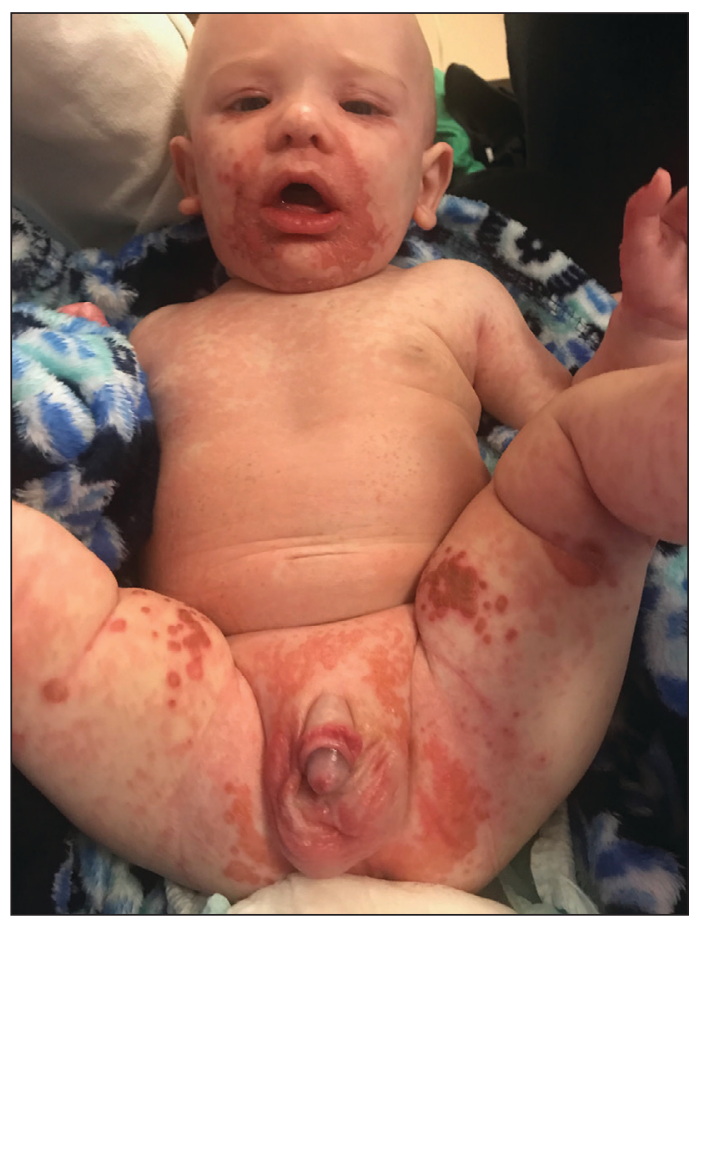

A 4-month-old infant boy presented to the pediatric hospital unit with a rash, fever, and failure to thrive. Prior to admission, the patient was treated for impetigo by a community dermatologist. After not responding to treatment, he was admitted and given intravenous acyclovir for 1 day by the pediatric hospitalist, and the dermatology service was consulted. The parents reported the patient had diarrhea for 1 month and a worsening rash over the last 2 weeks. The mother was breastfeeding. Physical examination revealed a fever (temperature, $38.9^{\circ} \mathrm{C}$ $\left[102^{\circ} \mathrm{F}\right.$ ) and an irritable infant whose growth curve had fallen from the 50th to 15th percentile since the 2-month well-baby examination. He had a fine, red, papular truncal rash with confluent plaques in a periorificial distribution that spared the inguinal skin folds, with some vesicles in a herpetiform presentation on the thighs as well as inflammation on the feet and hands. A complete blood cell count was within reference range, but the alkaline phosphatase level was low at $53 \mathrm{U} / \mathrm{L}$ (reference range, 72-307 U/L). A herpes simplex virus test was negative. A human immunodeficiency virus test and skin biopsy were performed.

\section{WHAT'S THE DIAGNOSIS?}
a. acrodermatitis enteropathica
b. biotin deficiency
c. cystic fibrosis
d. essential fatty acid deficiency
e. Langerhans cell histiocytosis

Ms. Cvancara is from the Department of Biology, George Fox University, Newberg, Oregon. Dr. Cvancara is from the Department of Dermatology, University of Washington, Seattle. 


\section{THE DIAGNOSIS: Acrodermatitis Enteropathica}

$\Lambda$ crodermatitis enteropathica (AE) was the presumptive diagnosis. Oral supplementation with zinc sulfate $3 \mathrm{mg} / \mathrm{kg} / \mathrm{d}$ was started immediately after a zinc level was ordered. A low zinc level of $15 \mu \mathrm{g} / \mathrm{dL}$ (reference range, 56-134 $\mu \mathrm{g} / \mathrm{dL}$ ) eventually was obtained. The lesions began to fade in 2 days along with return of normal feeding and disposition, and the patient was discharged with continued zinc supplementation.

Acrodermatitis enteropathica is an autosomalrecessive condition resulting in severe zinc deficiency caused by a defect of dietary zinc absorption in the duodenum and jejunum. ${ }^{1}$ It occurs in 1 in 500,000 individuals with no gender or racial predilection. It can be acquired or inherited. ${ }^{2}$ Recognition of clinical symptoms is essential due to potential death if untreated. Zinc is an important trace element required for the proper functioning of all cells and plays a large role in the metabolism of protein, carbohydrates, and vitamin A. Zinc deficiency impairs immune function, leading to bacterial infections. It also is a cofactor of numerous metal enzymes such as alkaline phosphatase, RNA polymerase, and numerous digestive enzymes. ${ }^{3}$

Our laboratory analysis revealed low alkaline phosphatase and zinc levels, which led to the diagnosis of $\mathrm{AE}$; unfortunately, these levels can be ambiguous. ${ }^{4}$ There are many causes of acquired zinc deficiency, including premature birth, low birth weight, zinc deficiency in maternal milk, exclusive parenteral nutrition, malabsorption syndromes such as Crohn disease and celiac disease, alcoholism, low calcium and phytate (cereal grain) diet, and kwashiorkor. ${ }^{5}$ The hereditary deficiency of zinc classically is known as AE and is caused by an autosomalrecessive mutation of the SLC39A4 gene on chromosome arm 8q24.3, which determines a congenital partial or total deficiency of the zinc transporter protein ZIP4. ${ }^{6}$

The clinical manifestations of acquired zinc deficiency and $\mathrm{AE}$ are similar and consist of 3 essential symptoms: periorificial dermatitis, alopecia, and diarrhea. Unfortunately, this clinical triad is complete in only $20 \%$ of patients with $\mathrm{AE}^{3}$ For example, our patient was too young for an alopecia determination. The disease typically presents with eczematous papules and sometimes vesiculobullous or pustular lesions located around perioral and acral areas (Figure 1) as well as the anogenital region (Figures 2 and 3). The severity of the skin lesions is variable. ${ }^{7}$ Our patient also presented with eczematous truncal papules on the chest (Figure 4). Acrodermatitis enteropathica usually presents during childhood after weaning. Along with the aforementioned skin findings, other symptoms in infancy can include diarrhea, mood changes, and anorexia. In school-aged children and toddlers, zinc deficiency is characterized by growth retardation, alopecia, weight loss, and recurrent infections.

In the differential diagnosis, the clinical presentation of biotin deficiency involves abnormalities of the hair, skin, nails, and central nervous system (eg, seizures, ataxia, deafness). ${ }^{8}$ Cystic fibrosis presentation depends on the multiorgan involvement, but neonates often present with failure to

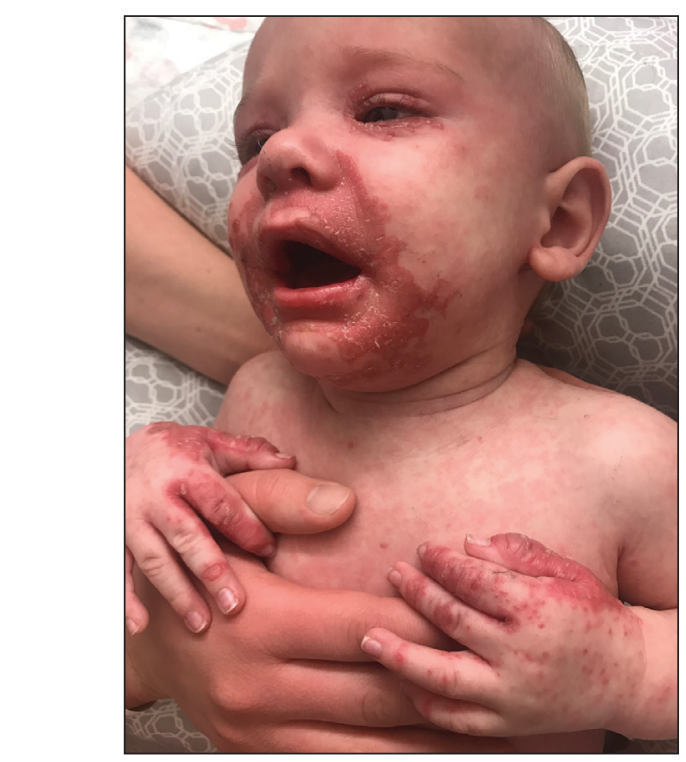

FIGURE 1. A periorificial vesicular rash on the hands and mouth characteristic of acrodermatitis enteropathica.

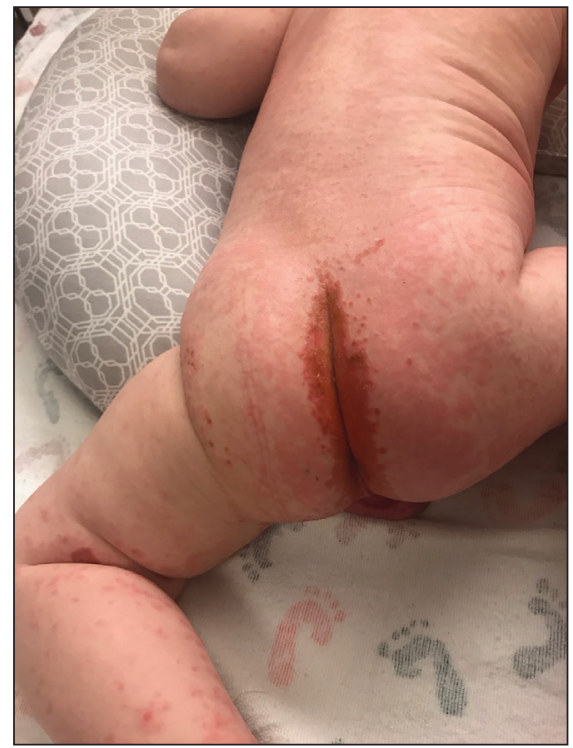

FIGURE 2. Anal involvement of acrodermatitis enteropathica 


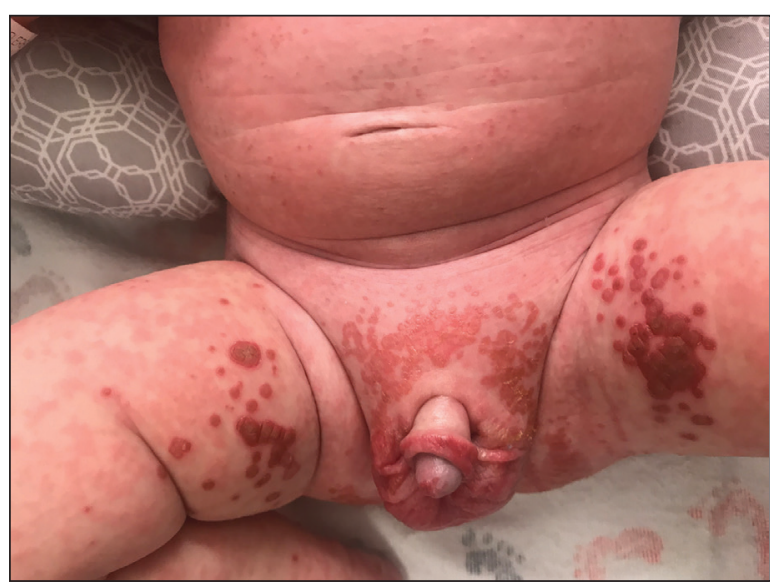

FIGURE 3. Genital involvement of acrodermatitis enteropathica.

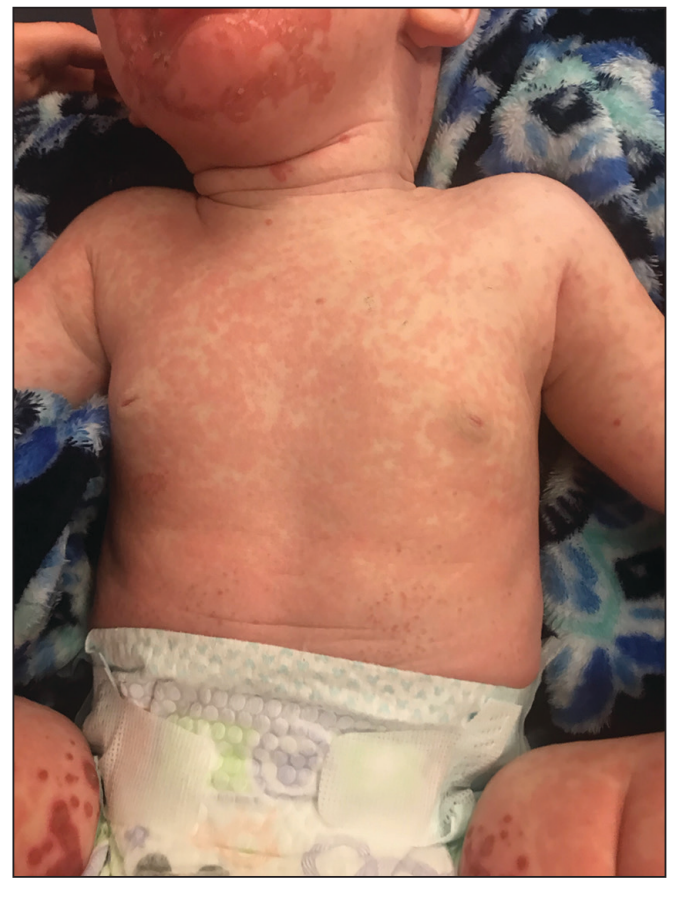

FIGURE 4. Fine eczematous truncal papules. thrive. ${ }^{9}$ Essential fatty acid deficiency presents clinically as dermatitis, alopecia, and thrombocytopenia, but a complete blood cell count with platelets was within reference range in our patient. ${ }^{10}$ Langerhans cell histiocytosis presents with perineal and postauricular lesions, but the skin biopsy did not confirm this diagnosis in our patient. ${ }^{11}$ Histopathologic examination of the buttock biopsy in our patient revealed nonspecific epidermal hyperplasia with acanthosis as well as clustered necrotic keratinocytes with vacuolization and parakeratosis.

Most clinicians who suspect $\mathrm{AE}$ treat with a therapeutic supplementation of zinc sulfate $3 \mathrm{mg} / \mathrm{kg} / \mathrm{d}$ while awaiting laboratory results. Acrodermatitis enteropathica is a rare condition, and early recognition of skin findings is important because misdiagnosis can lead to infections, malnutrition, and possibly death.

\section{REFERENCES}

1. Sehgal VN, Jain S. Acrodermatitis enteropathica. Clin Dermatol. 2000;18:745-748.

2. Van Wouwe JP. Clinical and laboratory assessment of zinc deficiency in Dutch children: a review. Biol Trace Elem Res. 1995;49:211-225.

3. Maverakis E, Fung MA, Lynch PJ, et al. Acrodermatitis enteropathica and an overview of zinc metabolism. J Am Acad Dermatol. 2007;56:116-124.

4. Van Wouwe JP. Clinical and laboratory diagnosis of acrodermatitis enteropathica. Eur J Pediatr. 1989;149:2-8.

5. Perafán-Riveros C, França LF, Alves AC, et al. Acrodermatitis enteropathica: case report and review of the literature. Pediatr Dermatol. 2002;19:426-431.

6. Kury S, Dréno B, Bézieau S, et al. Identification of SLC39A4, a gene involved in acrodermatitis enteropathica. Nat Genet. 2002;31:239-240.

7. Nistor N, Ciontu L, Frasinariu OE, et al. Acrodermatitis enteropathica: a case report. Medicine. 2016;95:E3553.

8. Gratias T. Biotin deficiency. Medscape website. https://emedicine .medscape.com/article/984803-overview. Updated October 22, 2018. Accessed October 15, 2020.

9. Sharma G. Cystic fibrosis. Medscape website. https://emedicine .medscape.com/article/1001602-overview. Updated September 28, 2018. Accessed October 15, 2020.

10. Morley JE. Essential fatty acid deficiency. Merck Manual website https://www.merckmanuals.com/professional/nutritional-disorders /undernutrition/essential-fatty-acid-deficiency. Updated January 2020. Accessed October 15, 2020.

11. Shea CR. Langerhans cell histiocytosis. Medscape website https://emedicine.medscape.com/article/1100579-overview. Updated June 12, 2020. Accessed October 15, 2020. 